\title{
Prise en charge médicale sur le long terme après chirurgie bariatrique
}

\author{
Dr JENNY DE FLINES ${ }^{\mathrm{a}}$, PrS ANDRE SCHEEN ${ }^{\mathrm{a}}$ et NICOLAS PAQUOT ${ }^{\mathrm{a}}$ \\ Rev Med Suisse 2019; 15: 1458-60
}

\begin{abstract}
L'efficacité de la chirurgie bariatrique est bien démontrée, mais le maintien d'un suivi médical est essentiel afin d'assurer une évolution satisfaisante sur le long cours. En effet, différentes problématiques peuvent survenir après une chirurgie bariatrique, dont l'évolution pondérale, parfois très variable: sous-poids, stabilisation précoce ou encore reprise pondérale progressive, qui nécessitent une mise au point souvent multidisciplinaire. L'apparition d'une ostéoporose précoce ou d'un dumping syndrome et le risque de carences en micronutriments sont des pathologies directement liées à la chirurgie bariatrique. En l'absence d'une prise en charge adéquate, elles peuvent devenir invalidantes et mettre à mal le succès de la chirurgie. Nous détaillerons le suivi médical conseillé à long terme des patients après chirurgie bariatrique.
\end{abstract}

\section{Long-term management after bariatric surgery}

The number of bariatric surgery interventions has increased over the past 10 years. The effectiveness of bariatric surgery is currently well demonstrated. However, the need to organize a careful medical follow-up of these patients is essential to ensure a positive evolution over the long term. In fact, weight change may be highly variable: excessive weight loss, early stabilization, progressive weight recovery in the middle or long term, all events that often require a multidisciplinary approach. The risk of osteoporosis, dumping syndrome and micronutrient deficiencies requires long-term medical monitoring. In absence of adequate management, such complications may hinder the success of surgery. This article describes the most important points of long-term medical follow-up of patients who underwent bariatric surgery.

\section{INTRODUCTION}

La chirurgie bariatrique a démontré son efficacité à long terme quant à l'évolution pondérale des patients qui souffrent d'obésité morbide. ${ }^{1}$ Elle a également prouvé ses effets bénéfiques sur les comorbidités liées à l'obésité comme le diabète de type 2, l'hypertension artérielle, l'apnée du sommeil. ${ }^{2}$ Le nombre d'interventions de chirurgie bariatrique n'a fait que croître ces dernières années. Les deux techniques opératoires principales sont la sleeve gastrectomy et le bypass gastrique. Les patients s'orientent de plus en plus vers ce type de prise en charge dès qu'ils répondent aux critères opératoires. On parle souvent, à raison, de l'importance du bilan multidisciplinaire préopératoire. Il est, en effet, essentiel de préparer au mieux

aservice de diabétologie, maladies métaboliques et nutrition, CHU Liège, 4000 Liège, Belgique

jenny.deflines@chuliege.be les patients à ce type d'intervention. De la même manière, il est important de connaître les différentes conséquences néfastes possibles de la chirurgie bariatrique à long terme afin de pouvoir répondre, de façon adaptée, aux questions et symptômes éventuels du patient. ${ }^{2}$ La mise en garde des patients et la prise en charge des pathologies classiques associées à ce type d'intervention font partie intégrante du suivi médical à long terme. Ceci doit être intégré, selon les besoins de chaque patient, dans une prise en charge multidisciplinaire reprenant des consultations en diététique, en psychologie et en chirurgie. L'objectif est d'assurer aux patients une amélioration maximale de la qualité de vie sur le long cours. ${ }^{1}$

\section{PROBLÉMATIQUE PONDÉRALE Sous-poids}

C'est une problématique relativement rare qui est la conséquence soit d'une diminution importante de la sensation de faim, soit d'une malabsorption intestinale secondaire à la chirurgie. En cas de perte de sensation de faim, la prise en charge consiste à donner des consignes d'enrichissements protéino-caloriques afin d'assurer des apports suffisants malgré la consommation de petits volumes alimentaires. Il est également important d'expliquer au patient la nécessité de suivre un régime fractionné ( 3 repas et 3 collations), sans tenir compte de l'appétit. En cas de malabsorption intestinale, le patient développe une symptomatologie digestive typique (diarrhée, ballonnement et douleur abdominale). Cette symptomatologie peut être la conséquence d'une diminution de la longueur de l'intestin grêle et/ou du retard de contact entre le bol alimentaire et les enzymes digestives (uniquement en cas de bypass) ou encore la conséquence d'un asynchronisme entre la vidange gastrique (accélérée) et la sécrétion des enzymes bilio-pancréatiques, secondaire au montage chirurgical (sleeve ou bypass). ${ }^{3}$ Dans cette situation, la prise en charge nutritionnelle reste indispensable (fractionnement, enrichissement protéino-calorique), mais doit souvent être couplée à la mise en place d'un traitement médicamenteux à visée symptomatique comme le lopéramide ou les analogues de la somatostatine. Dans le cadre de la gestion des troubles du transit, l'ajout d'enzymes pancréatiques peut aussi être utilisé dans certains cas afin d'améliorer l'absorption des protéines et des lipides contenus dans le bol alimentaire.

\section{Stabilisation précoce}

Cette évolution est définie comme une perte de poids inférieure à $60 \%$ de l'excès de poids à un an de l'intervention. Elle est, dans la grande majorité des cas, secondaire à une moins bonne adhésion du patient par rapport au suivi des 
conseils hygiéno-diététiques (régime alimentaire et pratique d'une activité physique adaptée). Dans certains cas, la réalisation d'une radiographie «œsophage-estomac-duodénum» et/ou d'une gastroscopie met en évidence une dilatation du néogastre: dans cette situation, l'avis du chirurgien est, bien entendu, conseillé. La prédisposition génétique du patient par rapport à la problématique pondérale joue certainement aussi un rôle dans la stabilisation précoce du poids. La prise en charge consiste en un suivi nutritionnel rapproché, associé à un suivi psychologique éventuellement. L'instauration d'un traitement médicamenteux de type biguanide (metformine) et/ou analogue du glucagon-like peptide-1 (GLP-1) doit être discutée au cas par cas. ${ }^{5}$

\section{Reprise de poids}

Comme expliqué dans le paragraphe précédent, le succès de la chirurgie bariatrique est très souvent évalué en fonction du pourcentage d'excès de poids perdu. On considère qu'une perte de poids supérieure à $60 \%$ de l'excès de poids à un an est un succès. ${ }^{4}$ On accepte également, selon les études, une reprise de poids de 5-10\% par rapport au poids minimal atteint dans les deux années qui suivent. Au-delà de ce pourcentage, on entre dans la véritable problématique de reprise de poids qui peut survenir à plus ou moins long terme par rapport à la chirurgie. Les facteurs favorisants de cette reprise de poids sont le nonrespect des consignes hygiéno-diététiques, l'arrêt de l'activité physique, l'instauration de certains médicaments tels que les corticoïdes, les traitements psychotropes au sens large, la dilatation du néogastre. La première étape de la prise en charge consiste, bien évidemment, à mettre en évidence l'origine de la reprise de poids afin de cibler au mieux les différentes options thérapeutiques. Si la problématique se situe au niveau du montage chirurgical, le patient devra être réorienté vers le chirurgien. Si elle est en lien avec l'instauration d'un traitement médicamenteux, la question de maintenir ce traitement devra se poser. En cas de reprise pondérale favorisée par le nonrespect des consignes hygiéno-diététiques, un suivi diététique rapproché, associé à la reprise d'une activité physique et, en fonction des besoins, un suivi psychologique seront indiqués. ${ }^{6}$ Linstauration d'un traitement médicamenteux pourra également être proposée. Comme décrit dans le paragraphe ci-dessus, les médicaments indiqués (metformine, analogues de GLP-1) devront être instaurés au cas par cas. ${ }^{5}$

\section{CARENCES EN MICRONUTRIMENTS}

Les carences en micronutriments sont une complication fréquente de la chirurgie bariatrique. ${ }^{7}$ Elles sont la conséquence d'une diminution drastique des ingesta, d'une tendance à la malabsorption (essentiellement dans le bypass gastrique), ou encore d'une intolérance à certains aliments. Les recommandations actuelles proposent une supplémentation quotidienne par un complexe vitaminique adapté à la chirurgie bariatrique durant les deux premières années postopératoires. Cette supplémentation doit être couplée à un suivi biologique semestriel. Au-delà de deux années postopératoires, ce suivi doit se poursuivre à raison d'un contrôle annuel minimum, à vie, et la supplémentation en micronutriments se fait alors «à la carte», en fonction des carences développées par le patient. ${ }^{8}$ Les plus fréquemment rencontrées concernent le fer, la vita- mine B12, l'acide folique, la vitamine $\mathrm{D}$ et le calcium. La problématique spécifique de la supplémentation phosphocalcique sera discutée ci-après. Des carences en vitamines B1, A et $\mathrm{E}$, ainsi qu'en zinc et sélénium, bien que plus rares, peuvent également apparaître. Il n'y a pas de recommandations spécifiques quant au type de supplémentation en micronutriments qui doit être donnée au patient après chirurgie bariatrique. Le plus important est d'assurer un suivi biologique régulier qui permettra de détecter les carences et d'adapter la supplémentation de façon individualisée. ${ }^{9,10}$

\section{Risque d'ostéoporose}

L'ostéoporose se caractérise par une perte excessive de la masse osseuse et de son architecture. Elle aboutit à une diminution de la résistance osseuse et à une augmentation du risque de fracture. Cette pathologie est déterminée par des facteurs génétiques, nutritionnels et environnementaux (notamment la sédentarité). Sur le plan nutritionnel, l'absorption intestinale du calcium dépend des ingesta et de la présence, en quantité suffisante, du métabolite actif de la vitamine D, la 1,25-dihydroxyvitamine D. La diminution de la calcémie entraîne une augmentation de la sécrétion de parathormone, ce qui a pour conséquence une balance osseuse négative. La chirurgie bariatrique, de type bypass gastrique ou sleeve gastrectomy, augmente le risque d'ostéopénie et d'ostéoporose suite au développement de carences en calcium et en vitamine D. Des supplémentations systématiques en calcium (citraté de préférence, pour optimiser l'absorption), à raison de $1200 \mathrm{mg} / \mathrm{jour}$, et en vitamine $\mathrm{D}$, à raison de $3000 \mathrm{UI} / \mathrm{jour}$, sont recommandées. L'objectif est d'atteindre une valeur de vitamine $\mathrm{D}$ sérique supérieure à $30 \mathrm{ng} / \mathrm{ml}$ dans les recommandations les plus récentes. Par ailleurs, en cas de bypass gastrique, la réalisation d'une ostéodensitométrie à deux ans de la chirurgie, et ensuite annuellement, est recommandée. Des études complémentaires pour analyser le réel impact sur le métabolisme osseux de la sleeve gastrectomy sont nécessaires, afin de proposer des recommandations concernant l'utilité de l'ostéodensitométrie dans le suivi des patients ayant opté pour ce type de chirurgie. .0,11 $^{1}$

\section{Dumping syndrome}

Le dumping syndrome est un effet secondaire fréquent et invalidant de la chirurgie bariatrique. ${ }^{12}$ Cette symptomatologie est bien connue chez les patients après bypass gastrique. Il ne faut cependant pas négliger l'existence de cette symptomatologie chez les patients ayant subi une sleeve gastrectomy. ${ }^{13} \mathrm{Il}$ existe deux formes de dumping syndrome: précoce et tardive.

La forme précoce survient dans les 30-60 minutes qui suivent l'ingestion du repas. Elle est caractérisée par l'arrivée rapide du bol alimentaire au niveau jéjunal. Ce contenu hyperosmolaire stimule la libération de peptides vasoactifs et d'incrétines qui entraîne, d'une part, des symptômes vasomoteurs (lipothymie, sudation, palpitations...) et, d'autre part, une symptomatologie digestive caractérisée par des douleurs abdominales, de la diarrhée et des ballonnements.

La forme tardive, survenant dans les deux à trois heures après le repas, résulte d'une absorption plus rapide du glucose associée à une réponse insulinique exagérée, ce qui entraîne des symptômes adrénergiques, d’une part, et neuroglucopéniques, 
d'autre part, typiques d'une hypoglycémie réactionnelle. ${ }^{14} \mathrm{La}$ prise en charge est essentiellement diététique. En effet, l'importance du fractionnement alimentaire, le respect d'une ingestion lente du repas et la limitation de la quantité de glucides (surtout ceux à index glycémique élevé) par repas sont essentiels. En cas d'hypoglycémies avérées, un traitement par acarbose, un inhibiteur sélectif des alpha-glucosidases intestinales, est indiqué en première intention ou, en cas d'échec, un traitement par analogue de la somatostatine peut également être essayé.

\section{CONCLUSION}

La chirurgie bariatrique nécessite un suivi médical à vie. En effet, le patient qui subit une chirurgie bariatrique est exposé à l'apparition de pathologies spécifiques en relation avec cette intervention. Les carences en micronutriments, à moyen et long termes, doivent être dépistées par la réalisation d'un bilan biologique annuel. Le dépistage de l'ostéoporose précoce est actuellement indiqué, surtout en cas de bypass gastrique. La problématique du dumping syndrome altère nettement la qualité de vie des patients et est également importante à reconnaître et à traiter. Enfin, le suivi médical à long terme de l'évolution pondérale est très important, à la fois pour les patients qui évoluent vers un sous-poids et une dénutrition, mais aussi pour les patients qui stabilisent précocement leur poids et pour ceux qui ont tendance à reprendre du poids sur

1 Sjöström L, Narbro K, Sjöström CD, et al. Effects of bariatric surgery on mortaltyin Swedish obese subjects. N Engl J Med 2007;357:741-52.

*2 Mantziari S, Duvoisin C, Demartines N, et al. Résultats à long terme ( $>/=10$ ans) après chirurgie bariatrique: revue de la littérature. Rev Med Suisse 2018;14:636-40.

3 De Flines J, Louis E, Paquot N. Exploration d'un syndrome de malabsorption digestive. Rev Med Liege 2018;73:526-32.

*4 Bonouvrie DS, Uittenbogaart M, Luijten AAPM, et al. Lack of standard definitions of primary and secondary (non)responders after primary gastric bypass and gastric sleeve: a systematic review. Obes Surg 2019;29:691-7. 5 Chang WW, Hawkins DN, Brockmeyer $J R$, et al. Factors influencing long-term weight loss after bariatric surgery. Surg Obes Rel Dis 2019;15:456-61. 6 Monpellier VM, Janssen IMC, Antoniou EE, Jansen ATM. Weight change after Roux en $Y$ gastric bypass, physical activity, and eating style: is there a relationship? Obes Surg 2019;29:526-33. 7 Favre Ferrario C, Mantziari S, Suter M. Recommandations de suivi des carences nutritionnelles apres chirurgie bariatrique. Rev Med Suisse 2019;15:626-30. ** 8 Dogan K, Homan J, Aarts EO, et al. Long term nutritional status in patients following Roux-en- $Y$ gastric bypass surgery. Clin Nutr 2018;37:612-7.

le long terme. En effet, le risque de réapparition des comorbidités liées au surpoids est grand et les enjeux en termes de santé publique sont, dès lors, très importants.

Conflit d'intérêts: Les auteurs n'ont déclaré aucun conflit d'intérêts en relation avec cet article.

\section{IMPLICATIONS PRATIQUES}

- La chirurgie bariatrique est une option thérapeutique validée chez le patient en obésité morbide (stade 3) ou avec comorbidités associées à une obésité sévère (stade 2)

- Un suivi nutritionnel à vie est conseillé afin de supplémenter en vitamines et oligoéléments si nécessaire et éviter l'apparition de carences

- Le dépistage et la prévention de l'ostéoporose doivent faire partie intégrante du suivi, essentiellement en cas de bypass gastrique

- Le dumping syndrome doit être pris en charge sur le plan diététique et éventuellement pharmacologique car il peut devenir très invalidant en termes de qualité de vie

- La gestion appropriée, essentiellement diététique et parfois aussi psychologique, de la reprise de poids, mais aussi du sous-poids, est importante à moyen et long termes

9 Thorell A. Clinical nutrition university: nutritional support after bariatric surgery. e-SPEN European e-Journal of Clinical Nutrition and Metabolism 2011;6:e96-100

10 Thibault R, Huber O, Azagury DE, Pichard C. Twelve key nutritional issues in bariatric surgery. Clin Nutr 2016;35:12-7. **11 Mechanick J, Youdim A, Jones DB, et al. Clinical practice guidelines for the perioperative nutritional, metabolic and nonsurgical support of the bariatric surgery patient- 2013 update: cosponsored by American Association of Clinical Endocrinologists, The Obesity Society, and American Society for Metabolic \& Bariatric Surgery. Obesity 2013;21:S1-27. 12 Emous M, Wolffenbuttel BHR, Totté E, van Beek AP. The short to mid term symptom prevalence of dumping syndrome after primary gastric bypass surgery and its impact on health-related quality of life. Surg Obes Relat Dis 2017;13:1489-500. *13 Ahmad A, Kornrich DB, Krasner H, et al. Prevalence of dumping syndrome after laparoscopic sleeve gastrectomy and comparison with Roux en $\mathrm{Y}$ gastric bypass. Obes Surg 2019;29:1506-13. *14 Scheen AJ. Diagnostic et évaluation d'une hypoglycémie chez le patient diabétique Rev Med Liege 2014;69:110-5.

\footnotetext{
* à lire

** à lire absolument
} 